\title{
Ergasilus urupaensis sp. n. (COPEPODA: ERGASILIDAE) das brânquias de Prochilodus nigricans AGASSIZ, 1829 (CHARACIFORMES: PROCHILODONTIDAE) da Amazônia Brasileira.
}

\author{
Jose Celso O. MALTA ${ }^{1}$
}

RESUMO - Ergasilus urupaensis sp. n. (Copepoda, Poecilostomatoida, Ergasilidae) é proposta. Os espécimens foram coletados dos filamentos branquiais de Prochilodus nigricans Agassiz, 1829 do Rio Urupá (afluente do rio Jiparaná), Estado de Rondônia, Brasil. A nova espécie tem uma seta forte, curva, pectinada e falciforme no primeiro exopodito, indicando relações com outras cinco espécies amazônicas. Esta espécie difere das outras no formato do cefalossomo, nas omamentações das pernas, das antenas e por apresentar um tamanho maior.

Palavras chaves: Copepoda, Ergasilus urupaensis sp. n., Poecilostomatoida, Ergasilidae, Amazônia.

Ergasilus urupaensis sp. $\mathrm{n}$. (Copepoda: Ergasilidae) from the gills of Prochilodus nigricans AGASSIZ, 1829 (Characiformes: Prochilodontidae) from the Brazilian Amazon.

ABSTRACT - Ergasilus urupaensis sp. n. (Copepoda, Poecilostomatoida, Ergasilidae) is proposed. The specimens were collected from the gill filaments of the freshwater fishes Prochilodus nigricans, from Urupá River, Rondônia state, Brazil. The new species has a curved pectinate seta on the first exopod that indicates relationship to five previously described Amazonian species. This species differs from others in the shape of cephalosome, ormamentation of the legs and antenna and by having a larger body.

Key-words: Copepoda, Ergasilus urupaensis sp. n., Poecilostomatoida, Ergasilidae, Amazon.

\section{INTRODUÇÀO}

O gênero Ergasilus Nordmann, 1832 é cosmopolita e ocorre tanto em água doce quanto salgada. Na região Neotropical são conhecidas 22 espécies, sendo que 12 são de água doce, encontradas principalmente nas brânquias de teleósteos. WILSON (1913) coletou a primeira espécie de Ergasilus parasitando elasmobrânquios e também o primeiro registro de ocorrência nas fossas nasais, o segundo registro de Ergasilus ocorrendo em fossas nasais foi o de VARELLA (1992).

A distribuição geográfica dos ergasilídeos Neotropicais consiste de duas espécies da Jamaica, uma da Costa Rica, duas da Colômbia, duas do Uruguai, uma da Argentina e 14 do Brasil. (KROYER, 1863 in: YAMAGUTI, 1963; WILSON, 1913; THOMSEN, 1949; SZIDAT, 1956; CRESSEY, 1970).

Das espécies brasileiras, uma é citada para o estado do Rio Grande do Sul, três para o de São Paulo, uma para a Paraíba, uma para o Pará, uma para Rondônia e sete para o estado do Amazonas (TIDD, 1942; PAIVA CARVALHO, 1955, 1962; MONTU, 1980; THATCHER, 1981a, 1981b, 1984; THATCHER \& ROBERTSON, 1982; THATCHER \& BOEGER,

Departamento de Biologia Aquática, Instituto Nacional de Pesquisas da Amazônia, Caixa Postal 478, Manaus, Amazonas, Brasil, CEP 69011-970. 
1983, 1984; THATCHER, BOEGER \& ROBERTSON, 1984; THATCHER \& PAREDES, 1984; MALTA \& VARELLA, 1986; BOEGER, MARTINS \& THATCHER, 1993).

A única espécie neotropical que é conhecido o desenvolvimento pós-embrionário e o macho de vida livre é $E$. bryconis Thatcher, 1981 (VARELLA, 1985).

Este trabalho descreve a $23^{\text {a }}$ espécie do gênero Ergasilus da América do Sul e a nona da região Amazônica.

\section{MATERIAL E MÉTODOS}

O material foi coletado no Estado de Rondônia, região noroeste do Brasil, durante o período de 28 de novembro de 1983 a 25 de setembro 1985 . Os peixes foram identificados, pesados e medidos. As brânquias e vísceras foram removidas e fixadas em formol $10 \%$. Os copépodos foram retirados dos filamentos branquiais utilizando finos estiletes, microscópio estereoscópio e transferidos para formol 5\%. Lâminas permanentes, com montagem total dos copépodos, foram preparadas usando o método de Thatcher, denominado "HYP" (publicado em Monoculus n.15 de novembro de 1987). Cada indivíduo foi retirado da solução aquosa (formol 5\%) e mantidos em álcool $70 \%$. A seguir, colocados em solução corante, Eosina e Orange-G. Posteriormente colocados em fenol e em seguida em salicilato de metila. Finalmente, foram montados em bálsamo do Canadá entre lâmina e lamínula e colocados em estufa à $70^{\circ} \mathrm{C}$.
Os desenhos foram feitos com o auxílio de câmara clara. As medidas foram obtidas com uma ocular micrométrica e expressas em micrômetros, são dadas as amplitudes seguidas pela média entre parênteses.

Os peixes foram depositados na Coleção Ictiológica do Instituto Nacional de Pesquisas da Amazônia, em Manaus.

Os tipos foram depositados nas coleções do Instituto Nacional de Pesquisas da Amazônia (INPA-CR), Manaus, Amazonas e Museu de Zoologia da Universidade de São Paulo, (MZUSP), São Paulo, São Paulo.

\section{Ergasilus urupaensis sp. n.}

Holótipo: fêmea (INPA-CR 614), dos filamentos branquiais de Prochilodus nigricans Agassiz, 1829; coletado no Rio Urupá (afluente do rio Jiparaná), próximo a cidade de Jiparaná (10'40'S; 61' 56'W), 3-X-1985, em lâmina. Parátipos: 8 fêmeas (INPA-CR $615 \mathrm{a}$ - f) e 2 fêmeas (MZUSP 10434 a e b) em lâmina dos filamentos branquiais de três Prochilodus nigricans da localidade tipo, dois coletados em 05-VI1984 e um em 03-X-1985. Todos coletados por J.C.O. Malta.

\section{Fêmea:}

Corpo (Fig. 1) comprimento total 833-958(895). Prossomo mais largo que o urossomo, largura do corpo 213296 (273).

Cefalossomo (Fig. 1) subovóide, margem anterior reta, alarga-se até sua metade, quando atinge a maior largura, esta largura se mantêm constante até o 


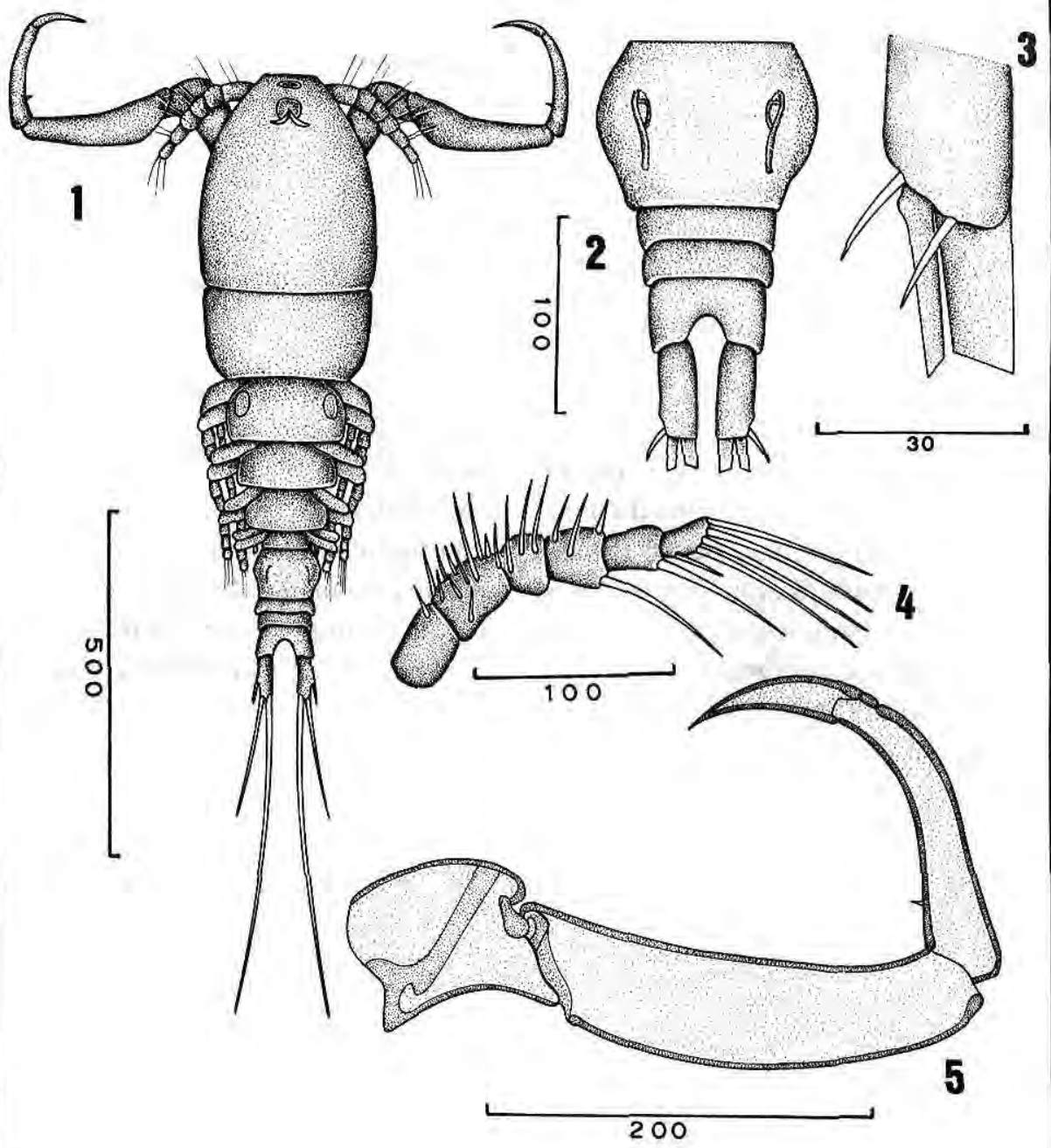

Figuras 1-5. Ergasilus urupaensis (fêmea) sp. n. 1 - vista dorsal. 2 - somito genital duplo, somitos abdominais e ramos caudais. 3 - ramo caudal (vista ventral). 4 - antênula. 5 - antena (medidas em micrômetros). 
terço final do cefalossomo onde afinase ligeiramente formando a segmentação com o primeiro somito pedígero. A partir deste ponto vai suavemente afilando-se até a margem posterior quando há um afilamento mais acentuado na margem distal do primeiro somito pedígero. Margens laterais arredondadas. Olho proeminente azul. Metassomo (Fig.1) com quatro somitos pedígeros (somitos 2, 3, 4 e 5), todos claramente separados e decrescendo suas larguras gradualmente na direção terminal.

Urossomo (Figs. 1, 2 e 3) menor que a metade do comprimento do prossomo e somito $6 \mathrm{com}$ as pernas natatórias rudimentares. Somito genital duplo (Figs. 1 e 2) suborbicular mais largo que comprido, comprimento 70-82(73), largura 80-97(86). Margens laterais arredondadas. Somitos abdominais (Figs. 1, 2 e 3), 1 e 2 sub-retangulares; somito abdominal 3 (anal) subretangular com a margem anterior reta e a posterior com as porções laterais projetando-se cerca do dobro do comprimento da região central do somito. Ramos caudais (Figs. 1, 2 e 3) subretangulares, ambos equipados com uma seta longa e uma curta e duas setas reduzidas.

Antênula (Fig. 4) comprimento 165-185(174) e maior largura 2240(29), cilíndrica, com seis segmentos, carregando 25 setas simples e com a fórmula setal: $1-11-3-4-1-5$. Antena (Fig. 5) com três segmentos e a garra. Segmento 1 sub-retangular e o menor, comprimento 112-135(122), largura 60-90(69); segmento 2 o maior e o mais robusto, comprimento 212245(233), largura 50-55(51); o segmento 3 com um pequeno espinho na margem proximal interna e um poro na margem distal externa, porção distal curva, comprimento 132-172(160) e largura 25-40(30); processo distal é uma forte garra curva, comprimento $80-90(85)$ e largura $12-17(15)$. A relação entre os segmentos incluindo a garra é $1,4: 2,7: 1,8: 1,0$.

Peças bucais (Fig. 6): mandíbula longa, com uma base robusta, estreita na parte proximal, sem ornamentações, alarga-se até cerca de um terço, na região mediana a margem anterior forma uma protuberância, neste ponto afilase cerca de um quarto da largura máxima, margem posterior, no terço distal origina-se o palpo mandibular, falciforme e com uma série de minúsculos dentes na margem posterior, o processo basal continua, afila-se e distalmente forma uma lâmina falciforme com minúsculos dentes na margem posterior. Maxílula pequena, em forma de placa, sem ornamentações. Maxila bisegmentada, processo proximal robusto, largo, sem ornamentações e fortemente preso ao cefalossomo, processo distal alongado, base mais larga, afilase suavemente, terminalmente falciforme com pequenas setas espiniformes circundando as margens anteriores. Maxilípedes ausentes.

Pernas (Figs. 7 - 10). Perna I (Fig. 7), basipodito com uma reentrância mediana na margem distal, endopodito com dois segmentos e exopodito com três. Segmentos do endopodito com uma fileira de espinhos na margem 

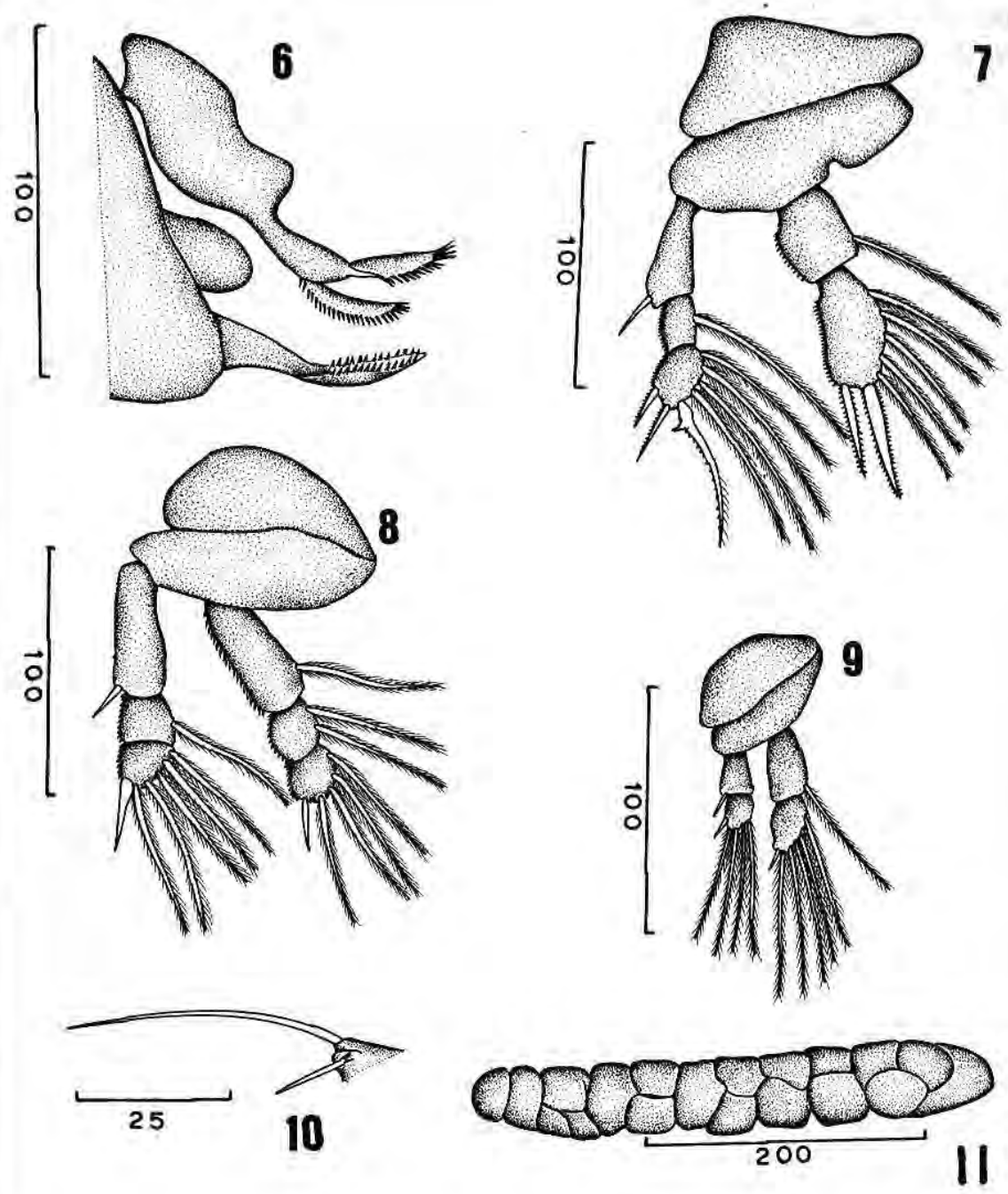

Figuras 6-11. Ergasilus urupaensis sp. n. (fêmea), 6 - peças bucais. 7 - perna I. 8 - perna II (= perna III). 9 - perna IV. 10 - perna V. 11 - saco de ovos (medidas em micrômetros). 
externa. Segmento 1 subquadrado, robusto e com uma seta; segmento 2 sub-retangular, com o dobro do tamanho do anterior, robusto, com cinco setas plumosas e duas fortes setas pectinadas terminais. Segmentos 2 e 3 do exdopodito com uma fileira de espinhos na margem externa. Segmento 1 subtriangular, o maior e com um espinho; segmento 2 sub-retangular, o menor e com uma seta; segmento 3 subquadrado com quatro setas, uma seta forte, curva, pectinada em forma de foice e dois espinhos pectinados.

Perna II (Fig. 8) semelhante à perna III com ambos os ramos de três segmentos. Todos segmentos do endopodito, com uma fileira de espinhos na margem externa. Segmento 1 subretangular, cerca de três vezes maior que os demais e com uma seta; segmento 2 com duas setas; segmento 3 com quatro setas e um espinho. Segmentos 2 e 3 do exodopodito, com uma fileira de espinhos na margem externa. Segmento 1 sub-retangular, cerca de três vezes maior que os demais e com um um espinho; segmento 2 com uma seta; segmento 3 com cinco setas e um forte espinho apical.

Perna IV (Fig. 9) com ambos os ramos de dois segmentos. Segmento 1 do endopodito sub-retangular e com uma seta; segmento 2 subovóide, com a margem posterior reta e com cinco setas e um espinho. Segmento 1 do exopodito com um espinho; segmento 2 com quatro setas e um espinho.

Perna V (Fig. 10) vestigial, consistindo de duas setas simples que se originam em uma papila, sendo uma seta cerca de cinco vezes maior que a outra.

Saco de ovos (Fig. 11) com duas séries de ovos que variam numericamente de doze a 23.

\section{Macho: Desconhecido.}

Etimologia: O nome específico deriva do nome do rio onde os exemplares foram coletados, Urupá.

\section{DISCUSSÃo}

Ergasilus urupaensis sp. n. tem uma seta pectinada, em forma de foice, no segmento terminal do primeiro exopodito, como as espécies: E. bryconis Thatcher, 1981; E. jaraquensis, Thatcher \& Robertson, 1982; E. hydrolycus, Thatcher, Robertson \& Boeger, 1984; E. callophysus Thatcher \& Boeger, 1984; E. holobryconis Malta \& Varella, 1986 descritas da região Amazônica, indicando haver relações entre elas.

Todas as medidas de $E$. urupaensis são muito semelhantes às de $E$. callophysus. Comparando-se o comprimento do cefalotórax de $E$. urupaensis com o de outras espécies de Ergasilus verifica-se que este corresponde a $48,0 \%$ do comprimento total, mesma proporção encontrada por THATCHER (1984) para E. versicolor Wilson, 1911. E. urupaensis tem maxílula reduzida e desprovida de espinhos como $E$. callophysus, $E$. hydrolycus e $E$. colomesus Thatcher \& Boeger, 1983.

E. urupaensis é a segunda maior espécie do gênero, da Região Neotropical, seu comprimento total variou de 
833-958 (895) micrômetros, só é menor que E. cyanopticus Paiva Carvalho, 1962 que mediu 1300 micrômetros. Apesar de comprida é uma espécie relativamente estreita pois apresentou a quarta menor largura do corpo.

A forma do cefalossomo de $E$. urupaensis é única entre os ergasilídeos neotropicais, sua margem anterior é reta, alarga-se até sua metade, quando atinge a maior largura, esta largura se mantêm constante até o terço final do cefalossomo onde afina-se ligeiramente formando a segmentação com o primeiro somito pedígero. A partir deste ponto, $\mathrm{O}$ primeiro somito pedígero vai suavemente afilando-se até a margem posterior quando há um afilamento mais acentuado na margem distal. Nenhuma das demais espécies conhecidas apresentam esta conformação. $E$. urupaensis é a única espécie que apresenta a antênula com a seguinte fórmula setal: $1-11-3-4-1-5$. A antena com um pequeno espinho na margem proximal interna, um poro na margem distal externa do terceiro segmento e com as seguintes proporções entre seus segmentos $1,4: 2,7: 1,8: 1,0$.

Comparando o somito genital duplo de E. urupaensis com o das demais espécies do gênero da região Neotropical, o formato e medidas são únicos, não se repetindo em nenhuma outra espécie.

O formato dos segmentos e os arranjos das ornamentações das pernas dão a E.urupaensis uma definição específica única tornando-a totalmente diferente das demais espécies. A perna $\mathrm{V}$, que é vestigial e consiste de duas setas, é a única que apresenta uma seta cerca de cinco vezes maior que a outra.

O número de ovos em $E$. urupaensis variou de $12-23$, esta é a menor quantidade encontrada em uma espécie neotropical.

Os índices parasitários de $E$. urupaensis foram: prevalência $26,0 \%$, intensidade variou entre 1 - 14 copépodos por peixe, intensidade média 4,0 e abundância 1,0 .

\section{Literatura Citada}

BOEGER, W. A; MARTINS, M.; THATCHER, V. E. 1993. Ergasilus hypophthalmi n. sp. (COPEPODA, POECILOS TOM A TOIDA. ERGASILIDAE) from the gill rakers of the amzonian catfishes, Hypophthalmus edentatus Spix and $H$. fimbriatus Kner. Rev. Brasil. Biol., 53(3):421-424.

CARVALHO, J. P. 1955 . Ergasilus xenomelanirisi sp. n. Parasito de PeixeRei - Xenomelaniris brasiliensi (QUOY e GAINARD), (Copepoda-CyclopoidaPisces-Mugiloidei). Bol. Inst. Ocean., (1-2):215-224.

1962. Ergasilus cyanopictus sp. n Parasito da Tainha Mugil cephalus (L.). Arq. Mus. Nac., 52:31-36.

CRESSEY, R. F, COLLETTE, B. B. 1970. Copepods and needlefishes: a study in host-parasite relationships. Fish. Bull., 68(3):347-432.

MALTA, J. C. O.: VARELLA, A. 1986. Engasilus holobryconis sp. n. parasita de Holobrycon pesu (MULLER \& TROSCHELL), um peixe da Amazônia Brasileira (COPEPODA: POE C I L OS T O M A T O I D A : ERGASILIDAE). Amazoniana, 10(1):4148. 
MONTU, M. 1980. Parasite copepods of Southern Brazilian fishes I- Ergasilus euripedesi n. sp. (Copepoda: Cyclopidea). Iheringia, 56:53-62.

SZIDAT, L. 1956. Uber die parasitenfauna von Percichthys trucha (CUVIER \& VALENCIENNES) Girard der Patagonischen gewasser und die bezienhungen des wirtsfishes und seiner Parasiten zur Palearktischen Region. Arch. f. Hydrobiol, 51(4):542-577.

THATCHER, V. E. 1981a. Os crustáceos parasitos de peixes da Amazônia Brasileira. 1. Ergasilus bryconis n. sp. (Copepoda: Cyclopoidea) da matrinchã (Brycon melanopterus). Acta Amazonica, 11(3):439-444.

1981b. Os crustáceos parasitos de peixes da Amazônia Brasileira. 2. Ergasilus leporinidis n. sp. (Copepoda: Cyclopoidea) das brânquias de Leporinus fasciatus (BLOCH). Acta Amazonica, 11(4)723-727.

1984. Ergasilus pitalicus, new species (Copepoda: Poecilostomatoida: Ergasilidae) a gill parasite of a cichlid fish from the $\mathrm{Pa}$ cific coast of Colombia. J. Crust. Biol., 4(3):495-501.

THATCHER, V. E; BOEGER, W. A. 1983. The parasitic crustaceans of fishes from the Brazilian Amazon. 4. Ergasilus colomesus n. sp. (Copepoda: Cyclopoidea) from an ornamental fish, Colomesus asellus (Tetraodontidae) and aspects of its patogenicity. Trans. Am. Microsc. Soc., 102(4):371-379.

1984. The parasitic crustaceans of fishes from the Brazilian Amazon. 9. Ergasilus callophysus n. sp. (Copepoda: Cyclopoidea) from Callophysus macropterus (LICHTENSTEIN). Proc. Helm. Soc. Was., 51(2):326-330.
THATCHER, V. E.; BOEGER, W. A.; ROBERTSON, B. A. 1984. The parasitic crustaceans of fishes from the Brazilian Amazon. 12. Ergasilus hydrolycus n. sp. (Copepoda: Poecilostomatoida) from Hydrolycus scomberoides (CUVIER). Amazoniana, 8(3):21-326.

THATCHER, V. E.; ROBERTSON, B. A. 1982 The parasitic crustaceans of fishes from the Brazilian Amazon. 3. Ergasilus jaraquensis n. sp. (Copepoda: Cyclopoidea) from the gills of Semaprochilodus insignis (SCHOMBURGK). Rev. Bras. Biol., v.42, n.3, p.515-519.

THOMSEN, R. 1949. Copépodos parásitos de los peces marinos del Uruguai. Com. Zool. Mus. Montivideo, 3(54): 1 - 41.

TIDD, W. M. 1942. A new species of Ergasilus (Parasitic, Copepoda) from a characinid fish of Brazil, Tr. Am. Micr. Soc. 62(1): $62-65$.

VARELLA, A. M. B. 1985. $O$ ciclo biológico de Ergasilus bryconis THATCHER, 1981 (Copepoda:Poecilostomatoida: Ergasilidae) parasita das brânquias do matrinxä, Brycon erythropterum (COPE, 1872) e aspectos de sua ecologia. Natal: Ed. Universitária. $100 \mathrm{p}$.

1992. Copépodos (Crustacea) parasitas das fossas nasais de peixes, coletados na região de Rondônia, Brasil. Tese de doutoramento, Instituto de Ciências Biológicas, Universidade Estadual Paulista, Rio Claro, São Paulo, 105p.

WILSON, C. B. 1913. Crustacean parasites of west Indian fishes and land crabes with descriptions of new genera and species. Proc. U. S. Nat. Mus., 44:189-277.

YAMAGUTI, S. 1963. Parasitic copepoda and branchiura of fishes. New York, John Wiley. 1104p. 